\title{
Caratteristiche idrodinamiche del lago di Sauris
}

\author{
P. Calor - G. Romualdi - M. C. Spadea
}

Ricevuto il 31 Marzo 1967

\begin{abstract}
"Un'improvvisa folata di vento rompe lo specchio verdazzurro del lago di Sauris, infrangendo un lucente legame fra le cose. $\Lambda l$ cader del vento, lo specchio si ricompone e l'acqua riprende il suo delicato tremulo colloquio con i boschi di abeti e le montagne intorno. A valle, la diga limita l'incanto: oltre la diga, l'asperrima ferrigna gola del Lumiei, orrida e possente. Lago di Sauris: sognante fragilità di motivi, aspirazioni vanenti in un brivido, specchio di cielo in bilico sopra l'abisso".
\end{abstract}

P. CaLor, Luglio 1951.

RIASSunto. - Vengono riassunti i dati sperimentali, registrati lungo le rive del lago di Sauris, in due diverse campagne di osservazioni: a bacino pieno e a bacino ridotto.

Il confronto fra i risultati raggiunti con quelli calcolati in un precedente lavoro, si traduce in una concordanza rimarchevole fra le osservazioni e i calcoli.

I valori dei periodi delle oscillazioni di nodalità superiore ad uno, essendo risultati sia nella teoria che nell'osservazione superiori a quelli relativi ad un lago con uniforme profondità, fecero ritenere applicabile al lago di Sauris la teoria corrispondente a bacini aventi sezione longitudinale a forma di triangolo rettangolo, con il cateto più corto in corrispondenza della diga. Sviluppata la teoria per questo caso speciale ed applicata al lago di Sauris, essa si è dimostrata perfettamente rispondente all'osservazione. Data la sua semplicità di applicazione, si ritiene preferibile studiare laghi analoghi con la nuova teoria.

SUMmarY. - Experimental results obtained along the banks of the Sauris's Lake, during two different groups of observations (full and reduced basin), are here resumed. 
The agreement between observed and precedent calculated data, is very remarkably. A new theory, moreover, is here showed and for the first time applied to an artificial lake.

As the values of the periods for $n$-nodal oscillations $(n>1)$ both in the theory and in the observation have been higher than these regarding a lake with even depth, we have applied the below explained theory to the Sauris's Lake.

It is considered a basin whose longitudinal section forms a right-angled triangle; the shorter cathetus figures the dam. agreed.

The theoretical and the observed results among themselves perfectly

The above mentioned theory, because of the easy applications, will be used for others similar lakes.

1. - Nel programma di lavoro sull'idrodinamica dei laghi italiani, tracciato da Caloi nel lontano $1947\left({ }^{2}\right)$, erano tacitamente comprese indagini su laghi artificiali che, oltre a presentare una batimetria di notevole precisione, consentono la registrazione delle oscillazioni libere a quote diverse del bacino lacustre, cosa pressochè impossibile - se non in misura ridottissima - in bacini naturali.

I'austero, bellissimo lago di Sauris offre queste possibilità. Esso si è formato in seguito allo sbarramento - mediante una diga alta 136 metri - dell'aspra, profonda valle del fiume Lumiei, presso le sue sorgenti sotto il monte Bivera $(2+73 \mathrm{~m})$.

A seconda dell'andamento delle stagioni e della richiesta di energia elettrica, il lago di Sauris puó essere a pieno invaso (quota $980 \mathrm{~m}$ circa) o a invasi più o meno parziali.

Nell'estate del 1962 fu eseguita una serie di misure limnografiche, a bacino pieno.

Nel 1965, per motivi legati all'esecuzione di alcune opere di consolidamento, il lago restó per molti mesi intorno alla quota 940 circa. $\mathrm{Ne}$ approfittammo per compiere, durante l'Agosto, diverse registrazioni limnografiche, lungo le rive del lago ridotto.

Si riassumono, qui di seguito, i risultati delle due accennate campagne di misure.

2. - Limnogrammi ottenuti durante l'Agosto 1962, a bacino pieno.

Le osservazioni limnografiche furono eseguite, successivamente ed alternativamente, nelle postazioni indicate con i numeri I, II, III, IV (Fig. 1). Si aveva a disposizione un solo limnografo (del tipo usato 


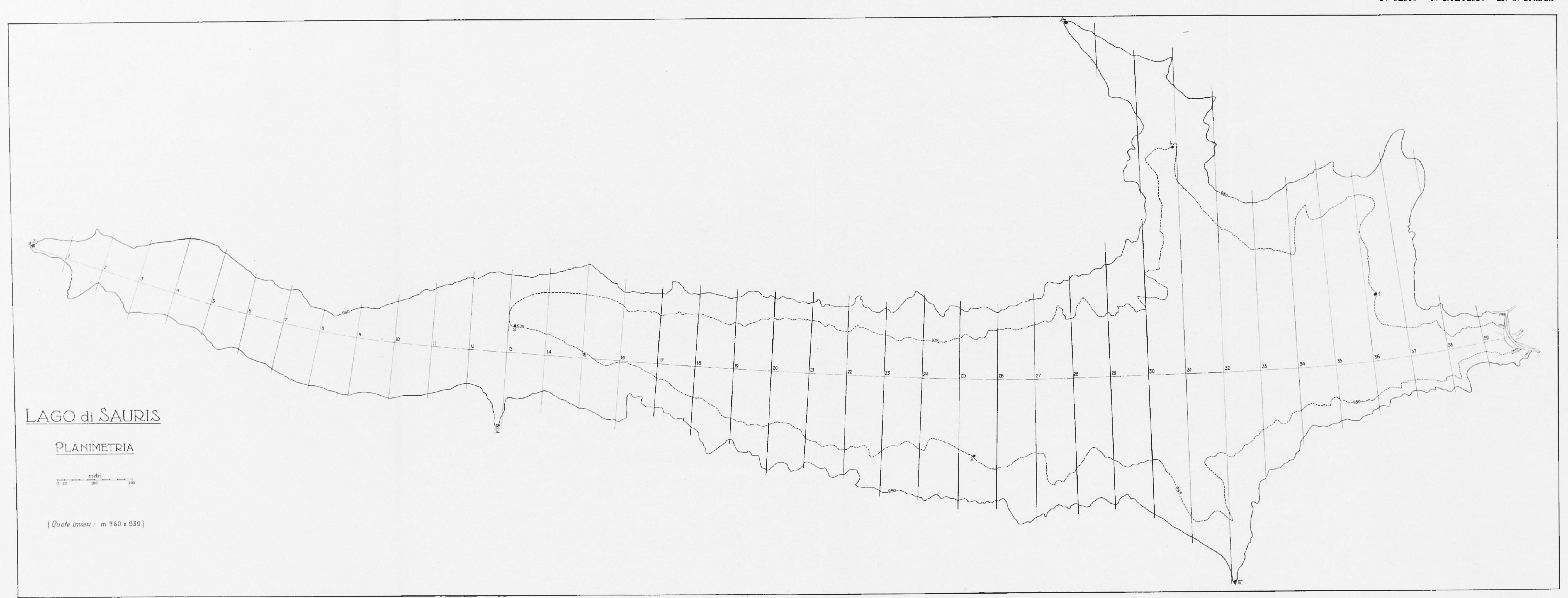

Fig. 1 - La linea tratteggiata delimita la superficie del lago ridoto (quota 939). 
in precedenti indagini su piccoli laghi), per cui non è stato possibile eseguire registrazioni contemporanee in due luoghi diversi.

Si è ritenuto superfluo eseguire misure continuate in corrispondenza della diga, nel punto cioè di massima profondità del lago. Un tentativo compiuto all'inizio delle osservazioni (20 Luglio 1962), ha dato infatti esito negativo, con oscillazioni di impercettibile ampiezza.

Il 30 Luglio il limnografo fu posto alla base della baia "verdazzurra " (II): fra le $10^{\text {h }}$ e le $11^{\text {h }}$ furono registrate chiare oscillazioni con periodo medio di 3 minuti circa (Fig. 2). Esse costituiscono esempi di binodali, registrate in II non ostante la vicinanza di un binodo. Sempre nella baia "verdazzurra", nella notte fra il 30 e il 31 , con il livello del lago in diminuzione, furono registrate lunghe serie di uninodali (periodo medio variante fra $5^{\mathrm{m}}, 9$ e $6^{\mathrm{m}}, 0$ ) (Fig. 3 ).

Il 6 Agosto 1962 il limnografo stava funzionando nella baia IV, la più ampia e più lunga di tutto il lago. La forma e le dimensioni della baia lasciavano prevedere la registrazione di sue oscillazioni proprie, cosa che si è verificata a più riprese, con particolare riguardo fra le $13^{\mathrm{h}}$ e le $16^{\mathrm{h}}$ (Fig. 4). Le oscillazioni hanno periodi fra $1^{\mathrm{m}}, 2$ e $1^{\mathrm{m}}, 4$, quali ci si doveva aspettare sulla base delle dimensioni della baia: esse si presentano ad impulsi singoli, successivi, ognuno esaurentesi in un solo ciclo: appunto come si verifica per bacini parziali, aperti su più ampia distesa d'acque.

Com'era naturale, i più lunghi periodi di registrazione furono quelli compiuti nella postazione I, all'estremo Ovest del lago. Tale estremo è raggiungibile da terra, solo dopo una faticosa complicata camminata lungo le rive. Il tragitto dalla località La Maina (presso la baia IV) veniva compiuto preferibilmente in motoscafo, anche per trasportare lo strumento.

Un primo esempio di registrazione, si ebbe il 7 Agosto, con una serie di uninodali, aventi periodi variabili fra $5^{\mathrm{m}}, 5$ e $6^{\mathrm{m}}, 0$, con prevalenza sui $6^{\mathrm{m}}$.

Un'altra serie di uninodali, di piccola ampiezza, fu ivi registrata il 16 Agosto; i periodi furono dell'ordine di $5^{\mathrm{m}}, 9$. Le registrazioni ottenute fra il 20 e il 22 Agosto - sempre all'estremo Ovest - presentano un particolare interesse. Si sono avute in quei giorni dei limnogrammi caratteristici, in cui i periodi più Iunghi risultano di circa $5^{\mathrm{m}}, 5$, alquanto inferiori quindi a quelli delle uninodali dell'intero lago che, in media, presentano periodi sui 6 minuti primi. Oltre al valore del periodo, anche il modo come si presentano ci porta ad escludere che si 
tratti di uninodali dell'intero bacino: esse, infatti, rivelano gli aspetti propri di movimenti oscillatori liberi di golfi aperti su bacini più ampli. Le registrazioni risultano di successioni di impulsi esaurentisi in un periodo (Figg. 5, 6 e 7 ): solo il persistere dell'azione perturbante permette il formarsi di più oscillazioni, ogni oscillazione essendo catturata come onda progressiva dal resto del lago, oltre la linea di bocca. Nel caso specifico, detta linea coincide all'incirca con la sezione $12^{2}$, come è facile provare con un breve calcolo.

Data la grande facilità della formazione di oscillazioni " locali ", interessanti la parte più occidentale del lago, l'estremo Ovest non costituisce la posizione più idonea per la registrazione delle uninodali pure; a questo scopo, si presta molto meglio la località "baia azzurra ", non commossa da oscillazioni parziali.

Anche alla base della baia III furono fatte alcune serie di registrazioni. In questa posizione si ottennero chiari esempi di uninodali pure (periodo di $6^{\mathrm{m}}$ circa). Oltre a "shelf-seiches" molto rapide (periodi inferiori al minuto primo), le più ampie oscillazioni ivi registrate sono caratterizzate da periodi dell'ordine di 1m,5 (Figgr. 8 e 9), che corrispondono a quelli delle trinodali.

\section{3. - Limnogrammi registrati durante l'Agosto 1965, con il bacino} a q. 939.

Numerose furono le registrazioni effettuate a lago ridotto, durante l'Agosto 1965. Qui si accennerà alle più interessanti.

Il limnografo venne sistemato successivamente, ed alternativamente, nelle postazioni segnate con i numeri 1, 2, 3, 4 (Fig. 1). A lago ridotto, gli spostamenti potevano essere eseguiti a piedi lungo le sponde, senza eccessiva difficoltà, verso tutte te postazioni, compresa la 2.

Qui si riferisce soltanto su parte delle registrazioni ottenute in località 1,2 , e 3 . Quelle avute in località 4, risultarono di scarso interesse.

A lago calmo, nella postazione 1 si ebbero lunghe serie di oscillazioni uninodali durante il 9 Agosto 1965. Il periodo medio risultò di $5^{\mathrm{m}}$ circa, con molte singole oscillazioni con periodi di $4^{\mathrm{m}}, 8-4^{\mathrm{m}}, 9$ (Fig. 10).

Alle $9^{\text {h } 30^{\mathrm{m}}}$ del 10 Agosto, sempre nella postazione 1 , il limnografo sorprese il lago animato da ampie oscillazioni uninodali (Fig. 11). Dopo le 10 si levó il vento, che andò via via rinforzando, raggiungendo il massimo fra le $12^{\mathrm{b}}$ e le $14^{\mathrm{h}}$, durante il contemporaneo infuriare di 


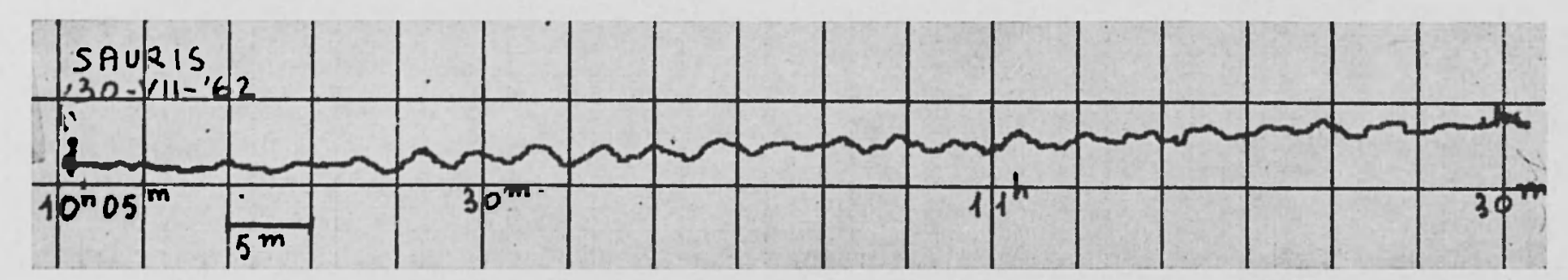

Fig. 2 - Esempi di binodali_registrate nella postazione II.

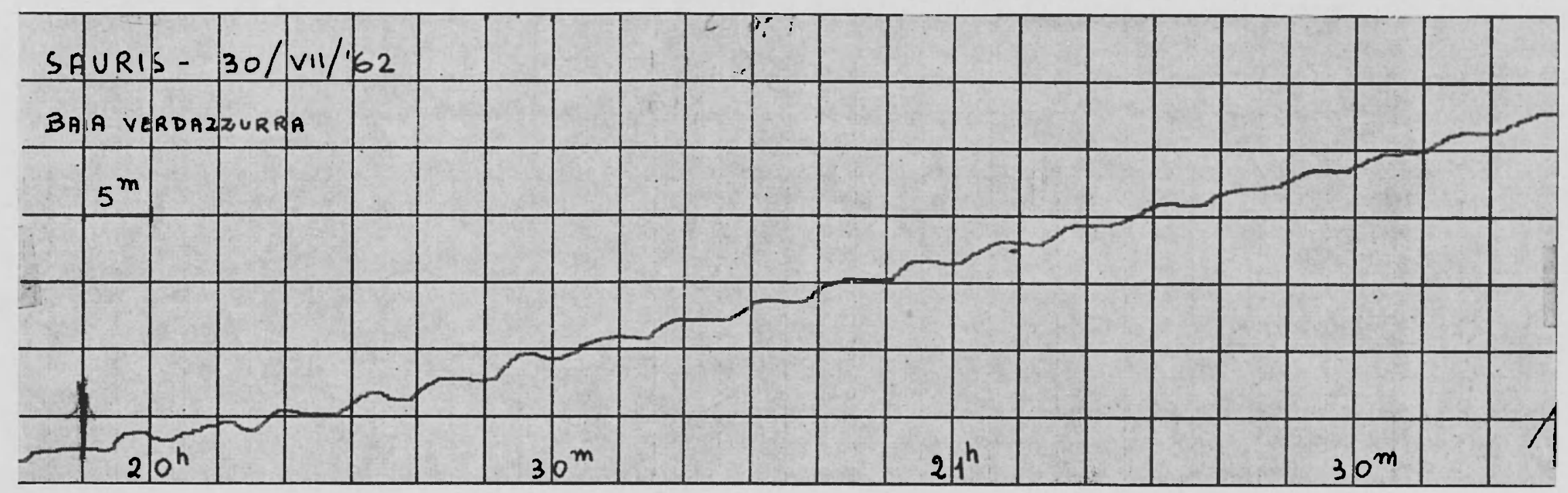

Fig. 3 - Uninodali a bacino pieno, registrate nella postazione II.

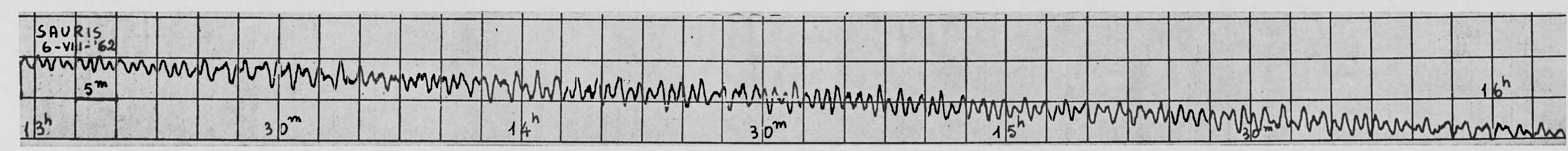

Fig. 4 - uscillazioni uninodali proprie della baia (post. IV): il nodo deve pensarsi in corrispondenza dell'apertura della baia sul resto del lago, nel quale le singole oscillaziour si propagano come onde progressive.

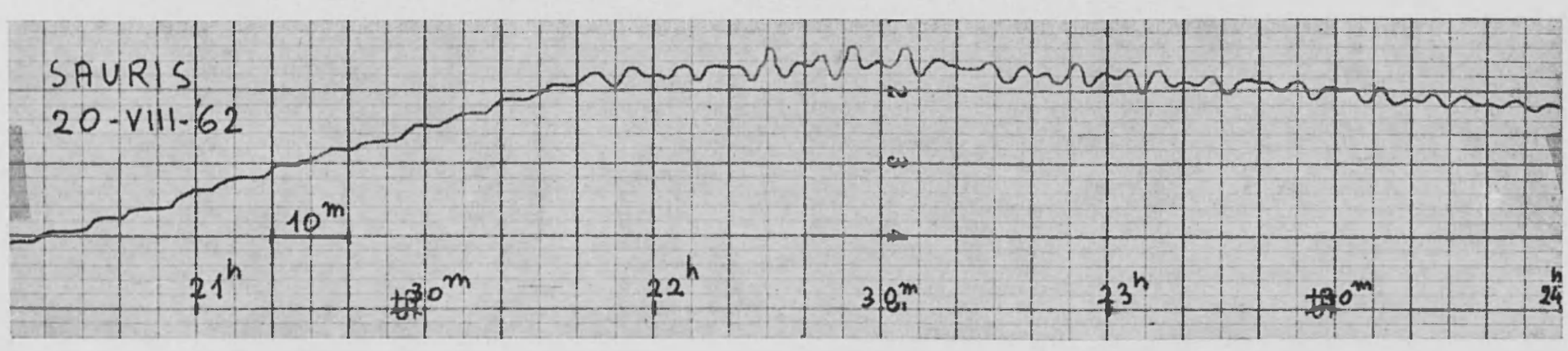
Fig. 5 - Registrazioni ottenute all'estremo Ovest del bacino pieno (post. I): esse costituiscono le uninodali della parte Ovest del lago,
avente la linea di bocca all'incirca in corrispondenza della sezione $12^{2}$.

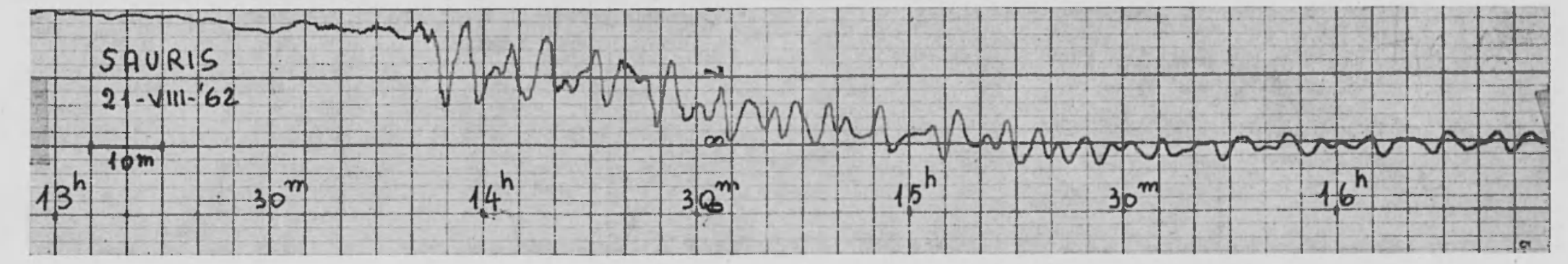

Fig. 6 - Uninodali della parte Ovest del lago (v. Fig. 5). 


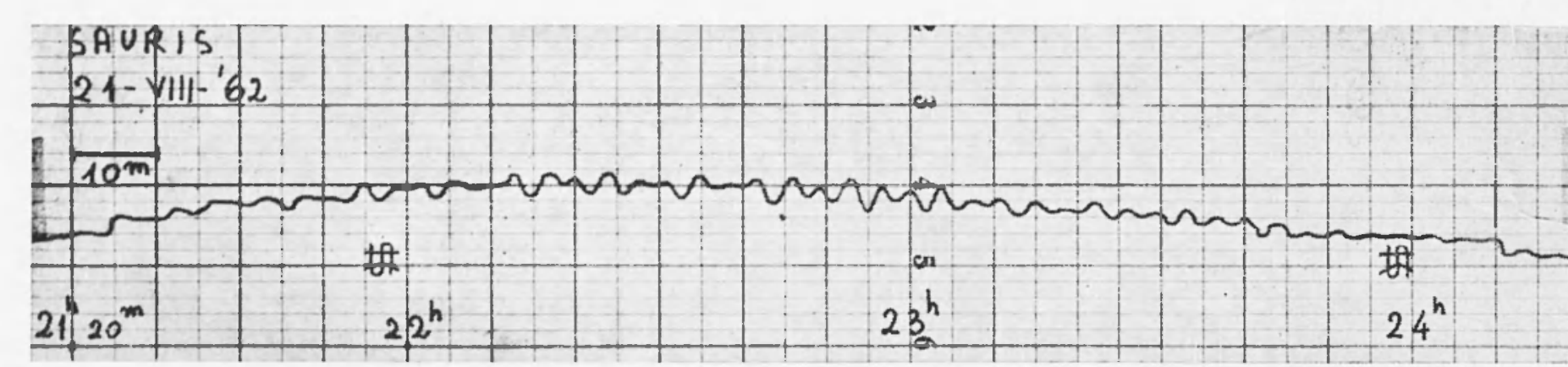

Fig. 7 - Uninodali della parte Ovest del lago (v. Fig. 5.

SAURIS $+15 / \mathrm{VIII/} / 62$

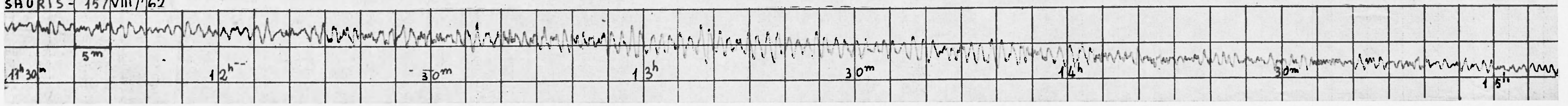

Fig. 8 - Registrazione di trinodali alla base della baia (post. III) - periodi dell' ordine di $\mathrm{l}^{\mathrm{m}, 5}$-, alternate a sesse da zoccolo (" shelf-seiches $\left.n\right)$.

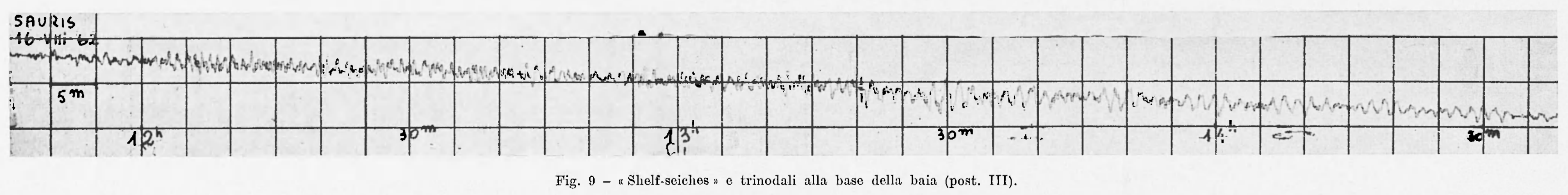

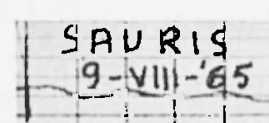

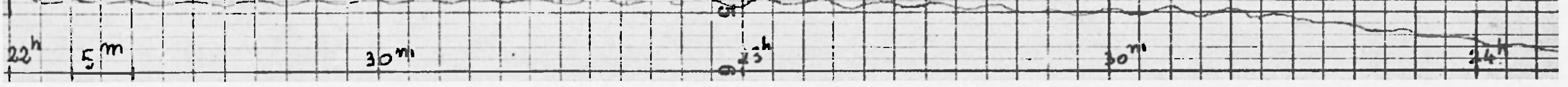




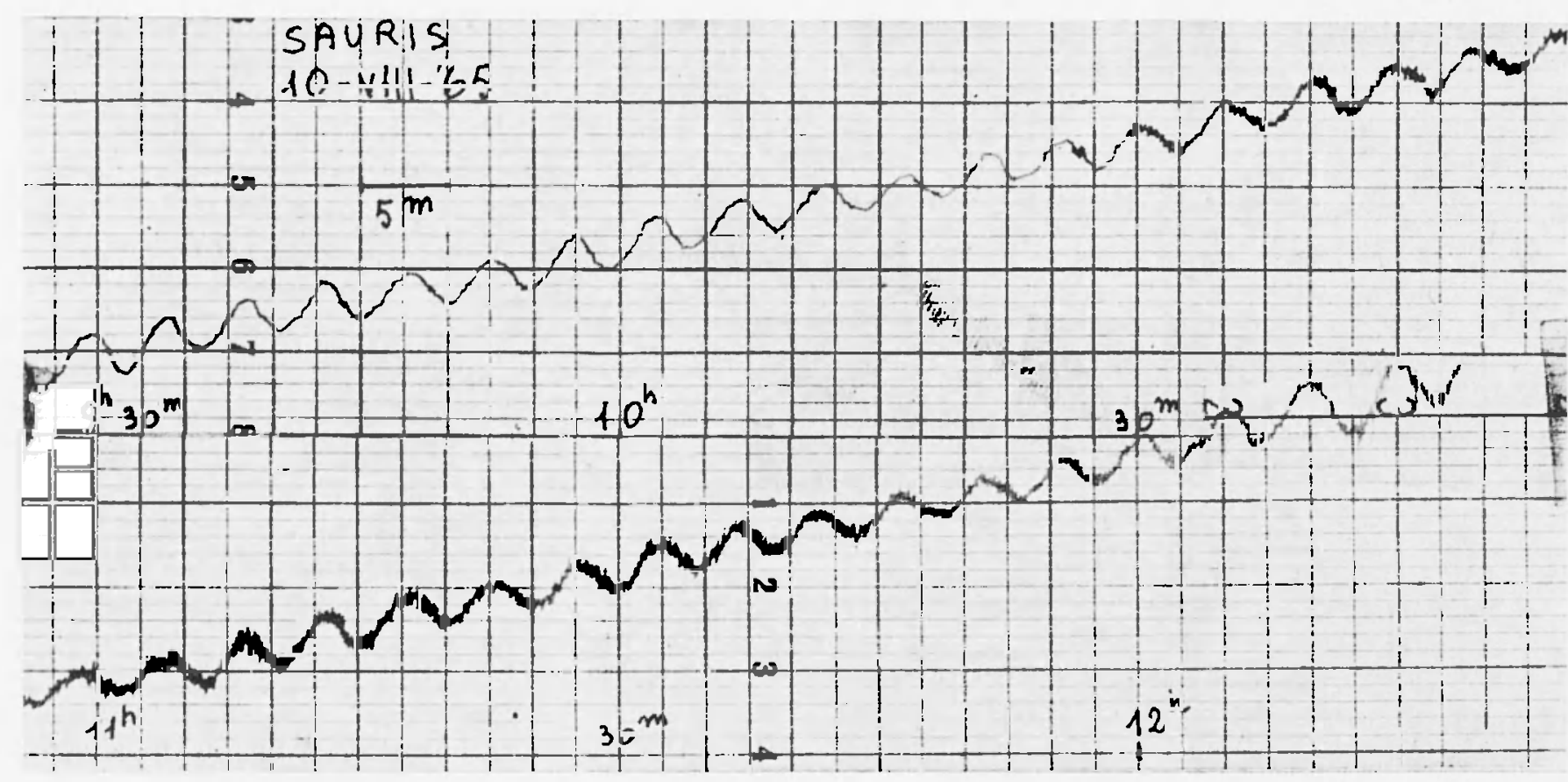

Fig. 11 - Uninodali nella postazione 1, con lago dapprima calmo, indi mosso da vento accompagnato da temporali.

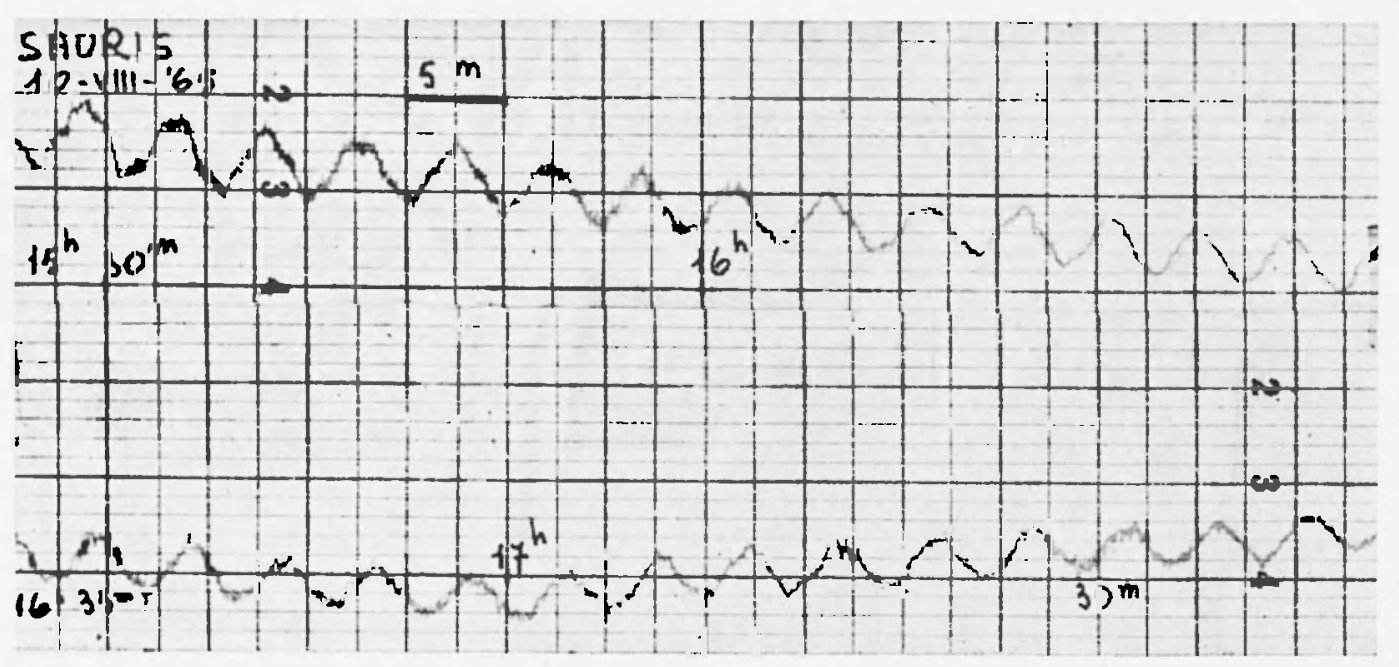

Fig. 13 - Uninodali nella postazione 1. 
P. Cator - G. Ronuatd - I. C. Spadea

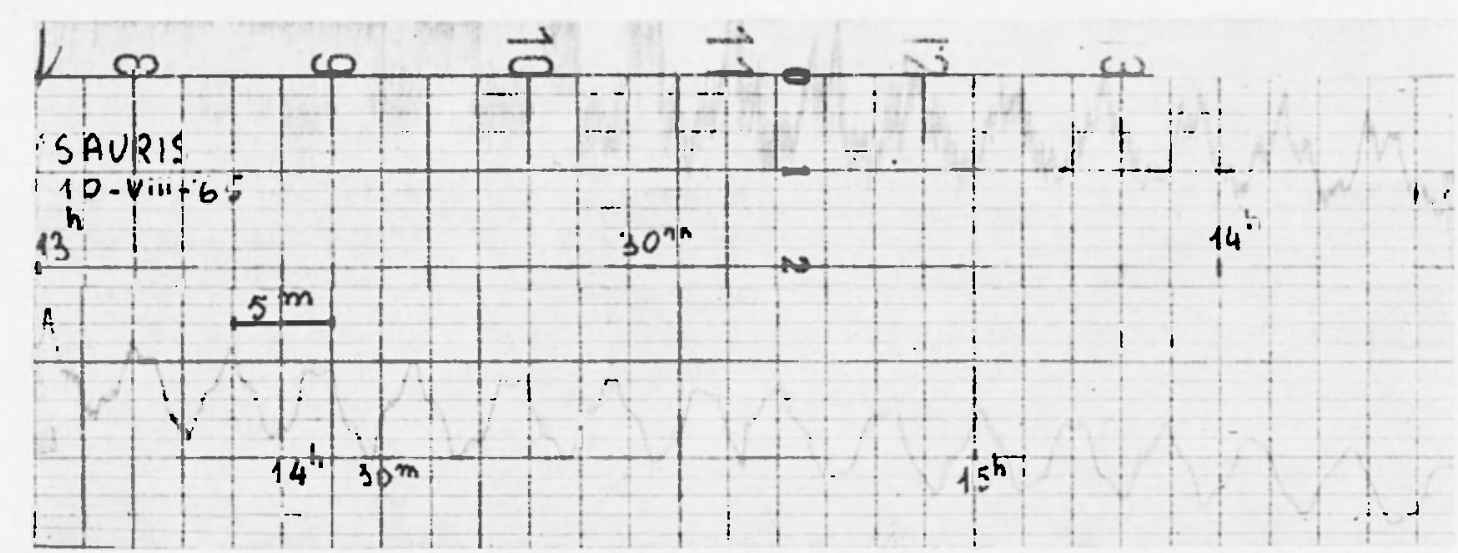

Fig. 12 - Uninodali nella postazione 1, durante e dopo il temporale, orlate da trinodali.

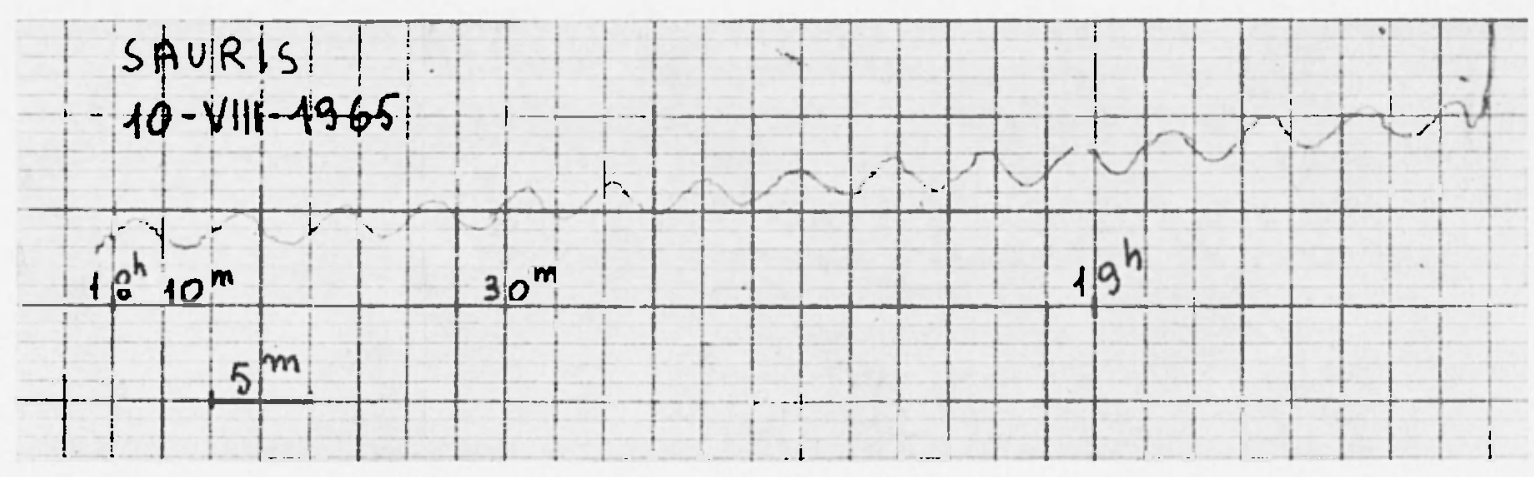

Fig. 14 - Uninodali nella postazione 1 (v. Figg. 11, 12, 13). 


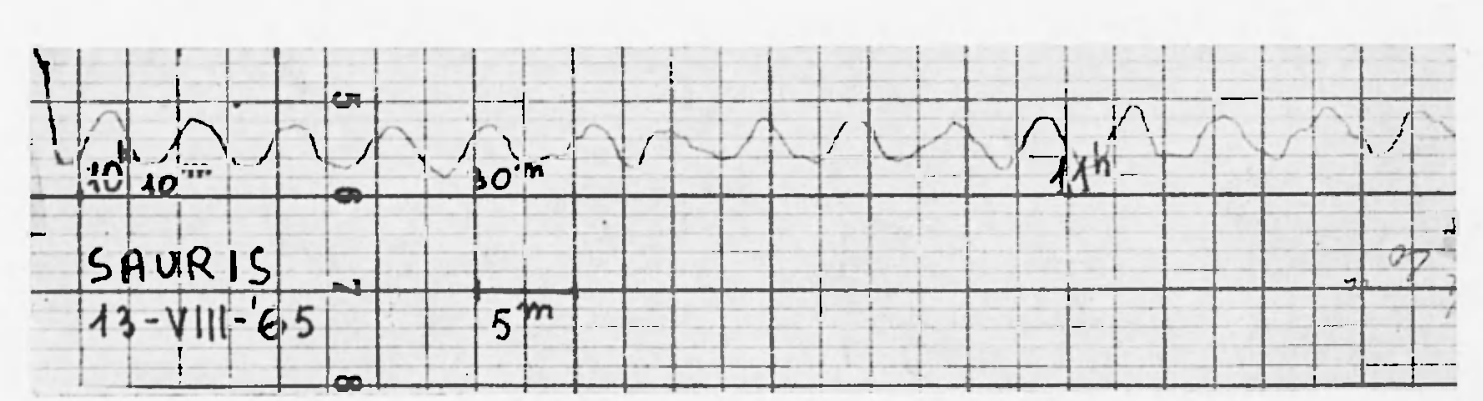

Fig. 15 - Uninodali del lago ridotto, registrate nella postazione 2.

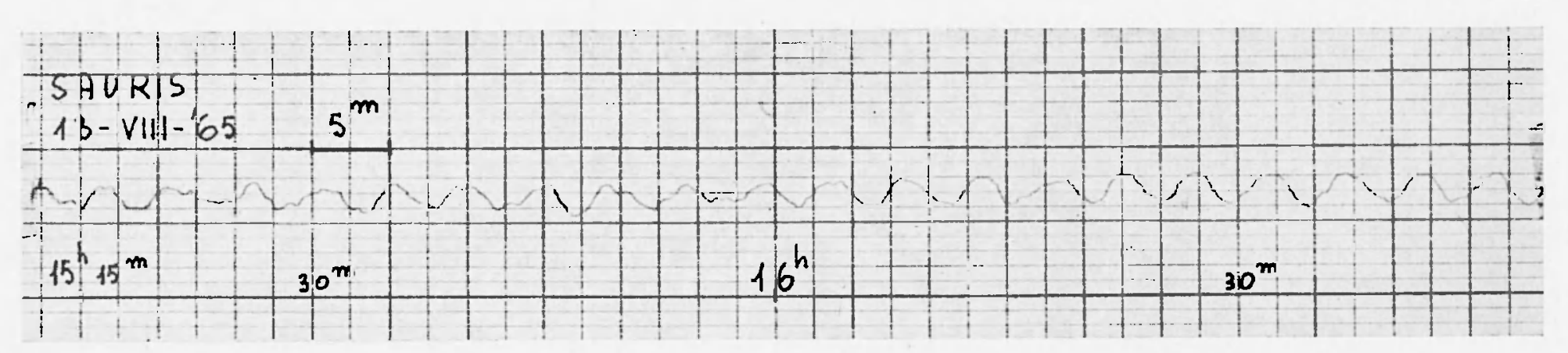

Fig. 16 - Uninodali del lago ridotto registrate nella postazione 2.

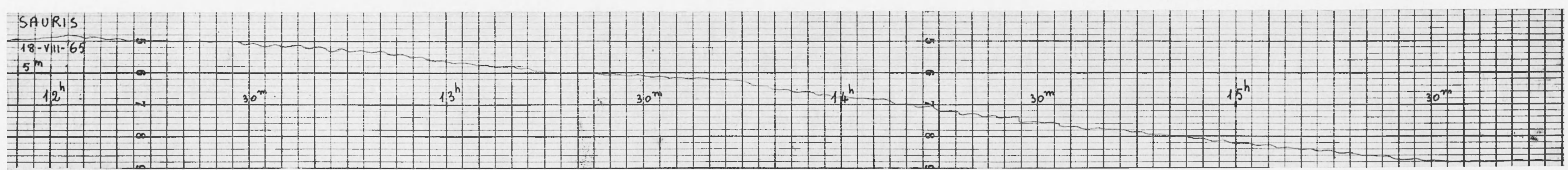


un temporale: il lago continuò ad oscillare sul ritmo di ampie uninodali, di un periodo medio fra $4^{\mathrm{m}}, 8$ e $5^{\mathrm{m}}, 0$, con prevalenza di quest'ultimo (Figg. 12 e 13).

Durante l'imperversare della burrasca, alle uninodali si sovrapposero oscillazioni di maggiore nodalità (binodali, trinodali, ...), che ne alterarono a lungo la forma. Verso notte, cessato il vento, le uninodali continuarono a susseguirsi pressochè pure (Fig. 14).

Il 13 Agosto il limnografo fu sistemato all'estremo Ovest, nella postazione 2. Anche qui si ebbero chiari esempi di onde uninodali (Figg. 15, 16), fra i quali predominano i periodi dell'ordine di $5^{\mathrm{m}}, 0$, anche se non mancano molte oscillazioni con periodi di 4,9 minuti primi.

Dalle esperienze compiute fino alla metà di Agosto, si era potuto arguire che, nel lago ridotto - a differenza di quanto si era verificato nel lago a pieno invaso - l'uninodale predominava incontrastata. Pertanto, si poteva sperare di registrare la binodale solo ponendo il limnografo nei pressi dell'uninodo. Ciò che si è fatto appunto nella seconda metà di Agosto, sistemando - a più riprese il limnografo sulla sponda destra, nella postazione 3. Ivi il 18 Agosto fu possibile registrare lunghe serie di binodali, piccole di ampiezza ma sufficientemente pure (Fig. 17): il loro periodo è risultato, in media, di $3^{m}, 1$.

Come osservazione complessiva, possiamo sottolineare il fatto che le uninodali osservate, coincidono praticamente con quelle calcolate. Per le binodali, l'accordo con la teoria - sempre buono - non può definirsi ottimo. Si osserva infatti che, sia per il lago completo sia per quello ridotto, il valore osservato supera (specie per il lago ridotto), in misura apprezzabile, quello calcolato.

Ne vedremo il motivo al n. 4.

4. - Nuova interpretazione idrodinamica dei moti liberi del lago di Sauris.

L'osservazione dei moti liberi del lago di Sauris, quale è stata riassunta nei numeri precedenti, permette di rilevare che l'oscillazione binodale si presenta con un periodo nettamente superiore alla metà del periodo dell'uninodale. Ciò ha suggerito la possibilità di un'altra interpretazione idrodinamica delle autoscillazioni del lago stesso. La riprova si poteva avere solo dopo la ricostruzione della sezione verticale longitudinale del lago, in corrispondenza della linea di valle. 
La Fig. 18 riproduce tale ricostruzione. Come ci aspettavamo, ai fini della teoria, il lago di Sauris può essere identificato con un bacino avente sezione longitudinale triangolare.

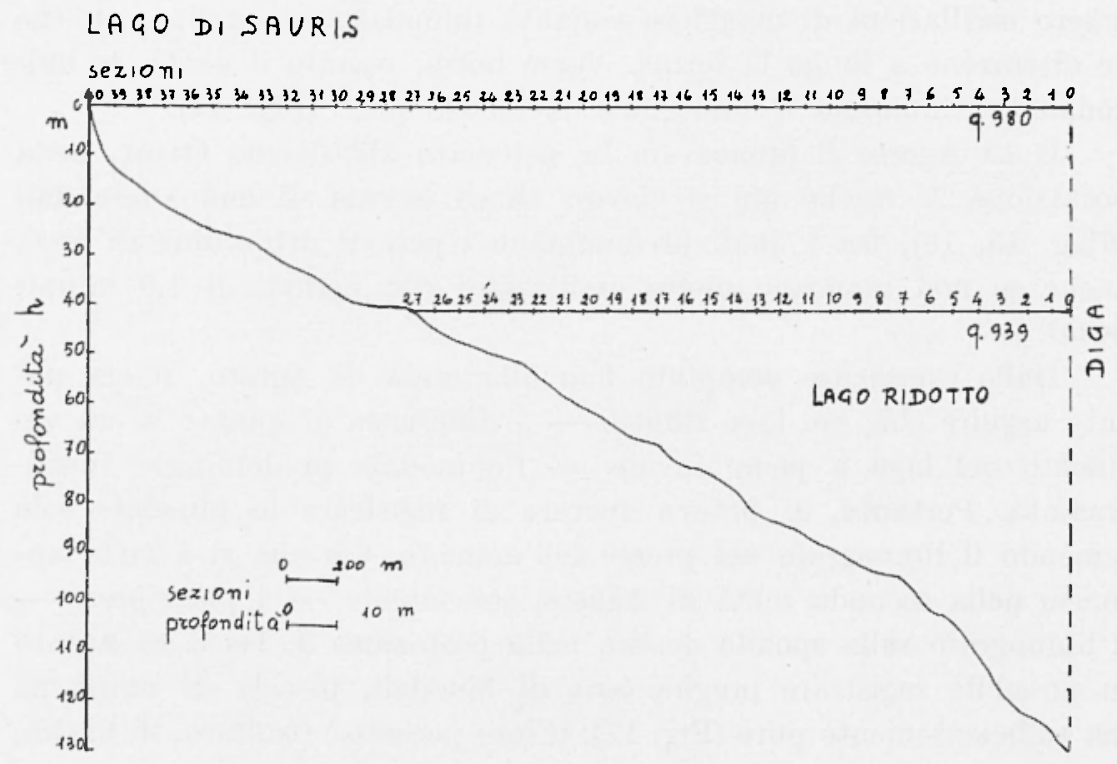

Fig. 18

Qui conviene fare un'opportuna digressione sui moti di laghi con fondo rettilineo, già studiati - nei loro aspetti generali — da G. Chrystal $\left(^{3}\right)$. Consideriamo un lago cosiffatto: se prendiamo l'origine delle $x$ in un punto dove la profondità è $h$, allora la legge della profondità diviene $h(x)=h(1-x / l)$, dove $l$ è una costante, positiva o negativar a seconda che il fondo del lago è inclinato verso l'alto o verso il basso nella direzione delle $x$ crescenti.

Abbiamo pertanto, con le abituali notazioni,

$$
\left\{\begin{array}{l}
\xi h(1-x / l)=u=P \sin n(t-\tau), \\
\zeta=-\frac{\partial u}{\partial x},
\end{array}\right.
$$

dove $P$ è determinatı dalla:

$$
\frac{d-P}{d x^{2}}+\frac{n^{2} P}{g h(1-x / l)}=0
$$


Trasformiamo la [2] ponendo:

$$
\omega=2 n l(1-x / l)^{1 / 2} /(g h)^{1 / 2} .
$$

E intanto:

$$
\frac{d^{2} P}{d x^{2}}-\frac{d^{2} P}{d \omega^{2}}\left(\frac{d \omega}{d x}\right)^{2}: \frac{d P}{d \omega} \frac{d^{2} \omega}{d x^{2}}
$$

$\mathrm{Ma}$

$$
\frac{d \omega}{d x}=-\frac{2 n^{2} l}{\omega g h}, \quad \frac{d^{2} \omega}{d x^{2}}=-\frac{4 n^{4} l^{\circ}}{g^{2} h^{2}} \cdot \frac{1}{\omega^{3}} .
$$

La [2] diviene pertanto:

$$
\frac{d^{2} P}{d \omega^{2}}-\frac{1}{\omega} \frac{d P}{d \omega}+P=0
$$

da cui, fatto $P=R \omega$, ed essendo:

$$
\frac{d P}{d \omega}=R+\omega \frac{d R}{d \omega}, \quad \frac{d-P}{d \omega^{2}}=2 \frac{d R}{d \omega}+\omega \frac{d^{2} R}{d \omega^{2}},
$$

consegue agevolmente:

$$
\frac{d^{2} R}{d \omega^{2}}+\frac{1}{\omega} \frac{d R}{d \omega}+\left(1-\frac{1}{\omega^{2}}\right) R=0
$$

La [3] ̀̀ un caso particolare dell'equazione di Bessel.

Se $J_{n}(\omega)$ e $Y_{n}(\omega)$ indicano funzioni di Bessel e di Neumann rispettivamente, la soluzione generale della [3] assume la forma ( $\left.{ }^{4}\right)$ :

$$
R=\mathrm{A} J_{1}(\omega)+B Y_{1}(\omega)
$$

Ne segue:

$$
\left\{\begin{array}{l}
\xi \omega=\left\{A J_{1}(\omega)+B Y_{1}(\omega)\right\} \sin n(t-\tau) \\
\zeta=\frac{h}{2 l}\left\{\frac{A}{\omega} \frac{\ddot{a}}{d \omega}\left(\omega J_{1}(\omega)\right)+\frac{B}{\omega} \frac{d}{d \omega}\left(\omega Y_{1}(\omega)\right)\right\} \sin n(t-\tau) .
\end{array}\right.
$$

Ora, per una delle proprietà fondamentali delle $J_{1}(\omega)$ e $Y_{1}(\omega)\left({ }^{5}\right)$, è:

$$
\frac{1}{\omega} \frac{d}{d \omega}\left(\omega J_{1}(\omega)\right)=J_{0}(\omega), \quad \frac{1}{\omega} \frac{d}{d \omega}\left(\omega Y_{1}(\omega)\right)=Y_{0}(\omega) .
$$


Perciò:

$$
\zeta=\frac{h}{2 l}\left\{A J_{0}(\omega)+B Y_{0}(\omega)\right\} \sin n(t-\tau) .
$$

Ai fini dell'utilizzazione delle formule [4] e [5], indicando con $j_{1}, j_{3}, j_{5}, \ldots$ le radici positive dell'equazione $J_{0}(z)=0$, e con $j_{2}, j_{4}, j_{6} \ldots$, le radici positive di $J_{1}(z)=0$ (escludendo la radice $j_{0}=0$ ), avremo approssimativamente - come si deduce dalle tavole $-j_{i}=2,405$, $j_{2}=3,832, j_{3}=5,520, j_{4}=7,016, j_{5}=8,651, j_{6}=10,173, j_{7}=11,792$, $j_{8}=13,323, j_{9}=14,931, j_{10}=16,471$, ecc.

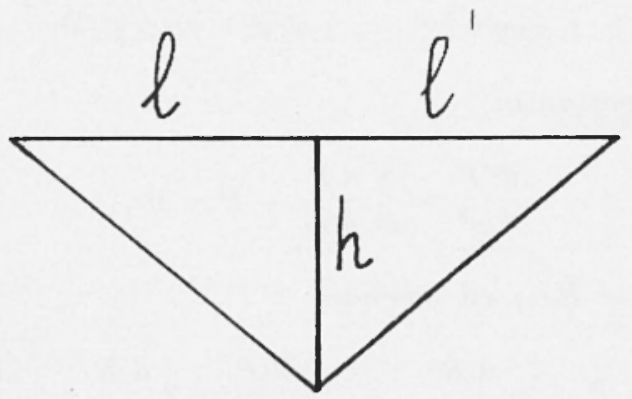

Fig. 19

Per grandi valori di $n, j_{n}=(2 n+1) \pi / 4$, approssimativamente: cioè, questa formula dà p. es. $j_{11}=18,064$ invece del valore corretto 18,071; così che l'errore dopo $n=11$ è minore dello $0,1 \%$. Ma questi grandi valori di $n$ a noi, evidentemente non interessano.

Per il problema all'esame, sarà bene considerare ora il caso di un lago simmetrico con pendenza costante fino ai due estremi (con sezione longitudinale avente quindi forma di un triangolo isoscele). Nella Fig. $19 \operatorname{sia} O A=O A^{\prime}\left(l=l^{\prime}\right)$.

Facciamo le posizioni:

$\omega=2 n l\left(1-\frac{x}{l}\right)^{1 / 2} /(g h)^{1 / 2} ; \quad \omega^{\prime}=2 n l^{\prime}\left(1+\frac{x^{\prime}}{l^{\prime}}\right)^{1 / 2} /(g h)^{1 / 2} ; \alpha=2 l /(g h)^{1 / 2}$.

Tenendo conto delle [4], [5], si giunge alle relazioni:

$$
\left\{\begin{aligned}
\omega \xi & =A J_{1}(\omega) \sin n(t-\tau) \\
\zeta & =\frac{h}{2 l} A J_{0}(\omega) \sin n(t-\tau) \\
\omega^{\prime} \xi^{\prime} & =A J_{1}\left(\omega^{\prime}\right) \sin n(t-\tau), \\
\zeta^{\prime} & =-\frac{h}{2 l} A J_{0}\left(\omega^{\prime}\right) \sin n(t-\tau) .
\end{aligned}\right.
$$


L'equazione dei periodi si spezza in:

$$
J_{o}(n \alpha)=0 ; \quad J_{1}(n \alpha)=0 .
$$

Perciò abbiamo:

$$
T_{\nu}=4 \pi l / j_{\nu}(g h)^{1 / 2} \quad(\nu=1,2,3, \ldots) .
$$

Si è visto che, per grandi valori di $\nu$, è approssimativimente $j_{\nu}=(2 v+1) \pi / 4$, per cui — per i grandi valori di $v-$

$$
T_{\nu}=16 l /(2 v+1)(g h)^{1 / 2} \text {. }
$$

E, quando $1 / 2 v$ è trascurabile, semplicemente:

$$
\begin{aligned}
T_{\nu} & =8 l / v(g h)^{1 / 2}= \\
& =4 L / v(g h)^{1 / 2}
\end{aligned}
$$

essendo $L$ la lunghezza del lago. Perció, col crescere della nodalità, i periodi delle sesse tendono sempre più a seguire la legge armonica $1,1 / 2,1 / 3,1 / 4, \ldots, \mathrm{e}$, in ultimo, a coincidere con $\mathrm{i}$ periodi di un lago uniforme di lunghezza doppia.

Se $\nu x_{r}$ denota la distanza da $O$ del $v$.mo suo nodo in $O A$, contando da $A$ verso $O$ ( $O$ essendo al centro del lago ed $A$ ad un estremo), e $\nu x_{r}$ ha significato analogo per i ventri, si trova:

$$
\begin{array}{ll}
1-\nu_{r} / l=\dot{j}_{2 r-1}^{2} / j_{\nu}^{2}=T_{\nu}^{2} / T_{2 r-1}^{2} & 1 \leqslant r \leqslant v \\
1-{ }_{\nu} x_{r} / l=\dot{j}_{2 r}^{2} / j_{\nu}^{2}=T_{\nu}^{2} / T_{2 r}^{2} &
\end{array}
$$

Perciò, se confrontiamo i diversi nodi della stessa sessa, le distanze dei nodi dall'estremità del lago, risultano inversamente proporzionali ai quadrati dei periodi delle sesse inferiori, di nodalità $d i$ spari. Se confrontiamo i nodi dello stesso ordine, per sesse differenti, le distanze dall'estremità riescono proporzionali ai quadrati dei periodi delle sesse corrispondenti.

Per un lago rettilineo completamente simmetrico, valgono i seguenti rapporti:

$$
\begin{array}{c|c|c|c|c|c|c|c|c}
T_{2} / T_{1} & T_{3} / T_{1} & T_{4} / T_{1} & T_{5} / T_{1} & T_{6} / T_{1} & T_{7 / T_{1}} & T_{8} / T_{1} & T_{9 / T_{1}} & T_{10} / T_{1} \\
\hline 0,6276 & 0,4357 & 0,3428 & 0,2779 & 0,2365 & 0,2040 & 0,1805 & 0,1609 & 0,1460
\end{array}
$$


Per lo stesso tipo di lago, la posizione dei nodi in una metà del lago è la seguente:

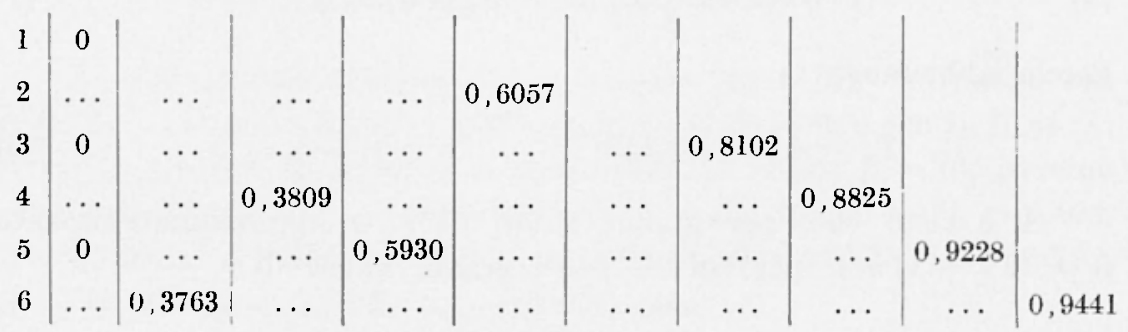

Mentre, per un lago di profondità uniforme, nella stessa metà la distribuzione dei nodi è:

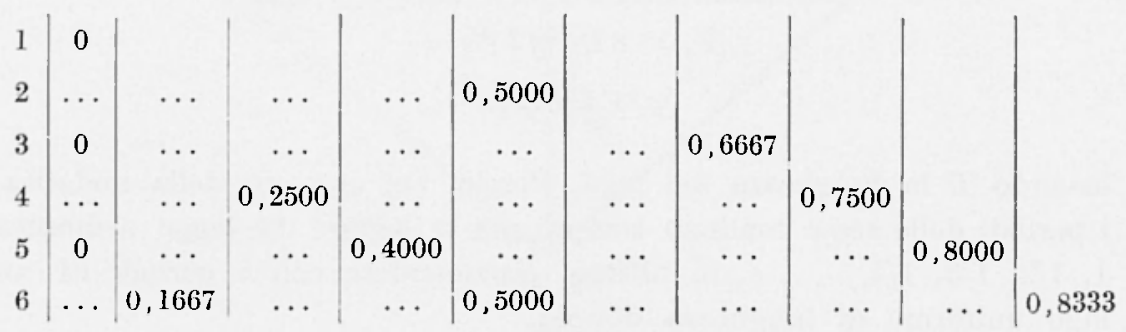

Veniamo finalmente al caso che più ci interessa, quello di un lago a fondo rettilineo inclinato in una sola direzione, affiorante in superficie (Fig. 20).

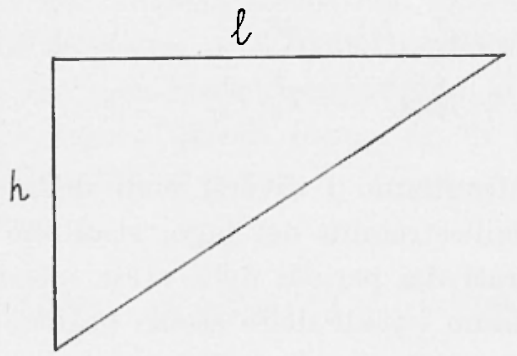

Fig. 20

Qui vanno considerate le oscillazioni libere di nodalità pari, prese in esame nel caso precedente:

$$
\left\{\begin{aligned}
\xi \omega & =A J_{1}(\omega) \sin n(t-\tau) \\
\zeta & =\frac{h}{2 l} A J_{0}(\omega) \sin n(t-\tau) .
\end{aligned}\right.
$$


L'equazione dei periodi è:

$$
J_{1}(n \alpha)=0
$$

così che:

$$
T_{\nu}=4 \pi l / j_{2 v} \quad(g h)^{1 / 2}
$$

I nodi sono, naturalmente, quelli delle autoscillazioni di nodalità pari nella metà destra del lago completo rettilineo simmetrico, prima considerato.

Analogamente a quanto si è ottenuto nel caso generale, valgono i seguenti confronti.

1) - Rapporti dei periodi in un lago rettilineo semincompleto:

\begin{tabular}{c|c|c|c|c|c|c|c|c}
$T_{2} / T_{1}$ & $T_{3} / T_{1}$ & $T_{4} / T_{1}$ & $T_{5} / T_{1}$ & $T_{6} / T_{1}$ & $T_{7 /} T_{1}$ & $T_{8} / T_{1}$ & $T_{9 /} T_{1}$ & $T_{10 /} T_{1}$ \\
\hline 0,5462 & 0,3767 & 0,2883 & 0,2327 & 0,1954 & 0,1684 & 0,1479 & 0,1319 & 0,1190
\end{tabular}

2) - Posizione dei nodi:

$$
\begin{array}{l|c|c|c|c|c|c}
\mathbf{1} & \ldots & \ldots & 0,6057 & & & \\
2 & \ldots & 0,3809 & \ldots & \ldots & 0,8825 & \\
3 & 0,3763 & \ldots & \ldots & 0,7056 & \ldots & 0,9441
\end{array}
$$

3) - Posizione dei nodi per profondità uniforme:

$$
\begin{array}{l|c|c|c|c|c|c}
1 & \ldots & \ldots & 0,5000 & & & \\
2 & \ldots & 0,2500 & \ldots & \ldots & 0,7500 & \\
3 & 0,1667 & \ldots & 0,5000 & \ldots & \ldots & 0,8333
\end{array}
$$

Applicazioni al lago di Sauris. Applichiamo la teoria esposta al lago di Sauris. Nel nostro caso si ha, per l'intero lago:

$$
\begin{aligned}
& l=m 3970 \\
& h=m 130,
\end{aligned}
$$

per cui l'equazione dei periodi [12] diviene:

$$
T_{\nu}=\frac{49863,2}{j_{2 \nu} \cdot 35,7}=\frac{1}{j_{2 \nu}} 1396,73 .
$$


Ricordiamo che $j_{2}=3,832 ; j_{4}-7,016 ; j_{6}=10,173, \ldots$ Pertanto sarà:

$$
\begin{aligned}
& \text { per l'uninodale: } T_{1}=364^{\mathrm{s}}, 5=6^{\mathrm{m}}, 1 \\
& \text { per la binodale: } T_{2}=199^{\mathrm{s}}, 1=3^{\mathrm{m}}, 3 \\
& \text { per la trinodale: } T_{3}=137^{\mathrm{s}}, 3=2^{\mathrm{m}}, 3 .
\end{aligned}
$$

Il risultato non potrebbe essere più soddisfacente, sia nei confronti dei valori ottenuti con il metodo di Hidaka, sia dei valori tratti dall'osservazione.

Per il lago ridotto, essendo:

$$
\begin{aligned}
& l=2670 m \\
& h=89 \mathrm{~m},
\end{aligned}
$$

la [12] diviene:

$$
T=\frac{1}{j_{2 \nu}} 1135,62 \quad(v=1,2,3, \ldots)
$$

La fondamentale e le due armoniche superiori assumono quindi, per il lago ridotto, i valori:

$$
\begin{array}{cll}
\text { Oscillazione } & \text { uninodale } & T_{1}=296^{\mathrm{s}}, 3=4^{\mathrm{m}}, 94 \\
\text { " } & \text { binodale } & T_{2}=161^{\mathrm{s}}, 9=2^{\mathrm{m}, 7} \\
\text { " } & \text { trinodale } & T_{3}=111^{\mathrm{s}}, 6=1^{\mathrm{m}}, 86 .
\end{array}
$$

Anche in questo caso, l'accordo fra teoria e osservazione appare ottimo.

Per quanto concerne l'andamento delle ampiezze, può servire la $2^{a}$ delle [11]. Basta calcolare l'andamento di $J_{0}(\omega)$ per le varie oscillazioni (ricordando che $h A / 2 l$ è una costante del lago). Allora, essendo:

$$
\omega=\frac{2 n l\left(1-\frac{x}{l}\right)^{1 / 2}}{(g h)^{1 / 2}}=n a\left(1-\frac{x}{l}\right)^{1 / 2},
$$

per l'uninodale si otterrà:

$$
\left.J_{o}(\omega)=J_{0}\left(n a \mid 1-\frac{x}{l}\right]^{1 / 2}\right)=J_{0}\left(j_{2 \nu}\left[1-\frac{x}{l}\right]^{1 / 4}\right),
$$


dove per $v=1, j_{2}=3,832$ e $x$ va inteso variabile da 0 ad $l$. Per la binodale $\left(\nu=2, \jmath_{4}=7,016\right)$ :

$$
J_{o}(\omega)=J_{o}\left(7,016\left[1-\frac{x}{l}\right)^{1 / 2}\right) \text {. }
$$

Per la trinodale $\left(\nu=3, j_{6}=10,173\right)$ :

$$
J_{\circ}(\omega)=J_{0}\left(10,173\left[1-\frac{a}{?}\right]^{1 / i c}\right) \text {. }
$$

L'applicazione delle [13], [14] e [15] al lago intero $(l=3970 \mathrm{~m})$ porta alla Tabella $\mathrm{I}$, mentre nel caso del lago ridotto $(l=2670 \mathrm{~m})$, i calcoli portano ai valori contenuti nella Tabella. II.

I grafici relativi costituiscono le Figg. 21-22 rispettivamente (*).

$\left(^{*}\right)$ Per confrontare i risultati ottenuti con quelli dedotti dall'applicazione del metodo di Hilaka generalizzato, fatta nel precedente lavoro ( ${ }^{1}$ ), abbiamo ritenuto opportuno calcolare l'andamento delle ampiezze della trinodale con il metodo stesso, la cui applicazione era stata limitata alla binodale.

Nel caso $m=2$, com'è stato provato da uno di noi (vedi $\left({ }^{2}\right)$, Parte I, pag. 19), l'equazione dei nodi è:

$$
4 \frac{A_{2}}{A_{n}} z^{3}+?\left(\begin{array}{ll}
\frac{A_{1}}{A_{0}} & \frac{A_{2}}{A_{0}}
\end{array}\right) \approx^{2}: \simeq\left(\begin{array}{ll}
1 & \frac{A_{1}}{A_{0}}
\end{array}\right) z-1=0 .
$$

Tenendo conto dei valori di $\lambda$ corrispondenti alle trinodali del lago intero e di quello ridotto, con il metodo esposto nel lavoro citato, si ottengono per i rapporti $A_{1} / A_{0}$ e $A_{2} / A_{0}$ i valori:

$$
\begin{cases}\frac{A_{1}}{A_{0}}=-8,20330 ; & \left.\frac{A_{2}}{A_{0}}=12,05564 \text { (per il lago a quota } 980\right) \\ \frac{A_{1}}{A_{0}}=-4,77846 ; & \frac{A_{0}}{A_{0}}=3,52873("\|"\| " 939) .\end{cases}
$$

Con queste coppie di valori, risolta la $(\alpha)$ nei due casi con il metodo delle approssimazioni successive, si ottiene:

$$
\begin{aligned}
& \left({ }_{3} z_{1}=0,06934{ }_{3} z_{2}=0,35991 \quad{ }_{3} z_{3}=0,83109\right. \text { (lago a quota 980) } \\
& \text { ( }{ }_{3} z_{1}=0,07261 \quad{ }_{3} z_{2}=0,38227 \quad{ }_{3} z_{3}=0,83993(\text { " " " 939). }
\end{aligned}
$$

I nodi cadono quindi, per il lago a q. 980, fra le sezioni 7-8, 22-23, 34-35, alle distanze di ca. $\mathrm{km} 0,79,2,3,3,4$ rispettivamente, dall'estremo Ovest 


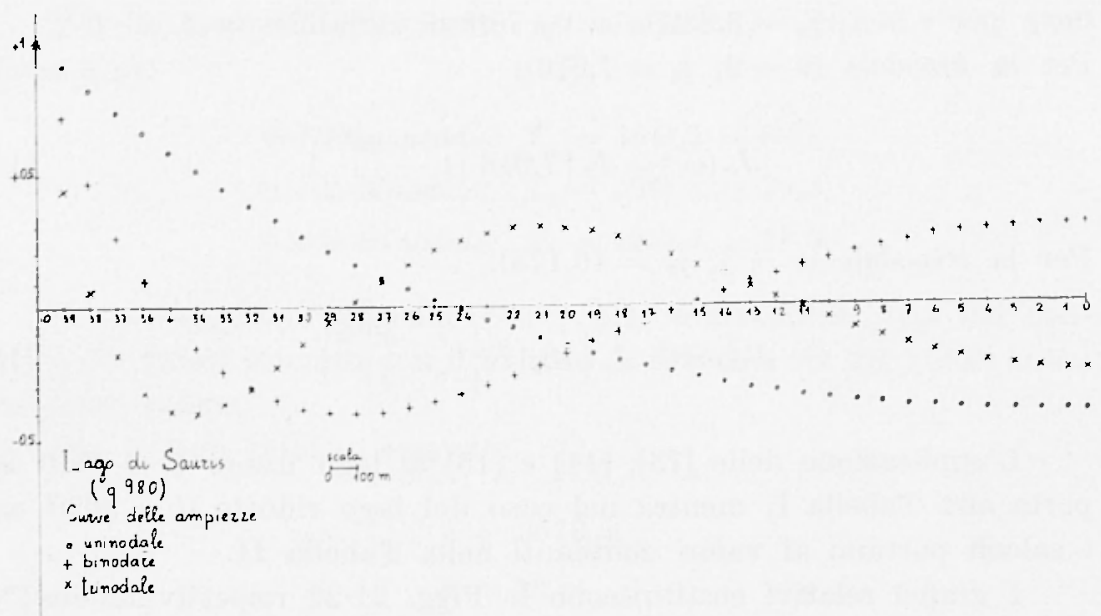

Fig. 21

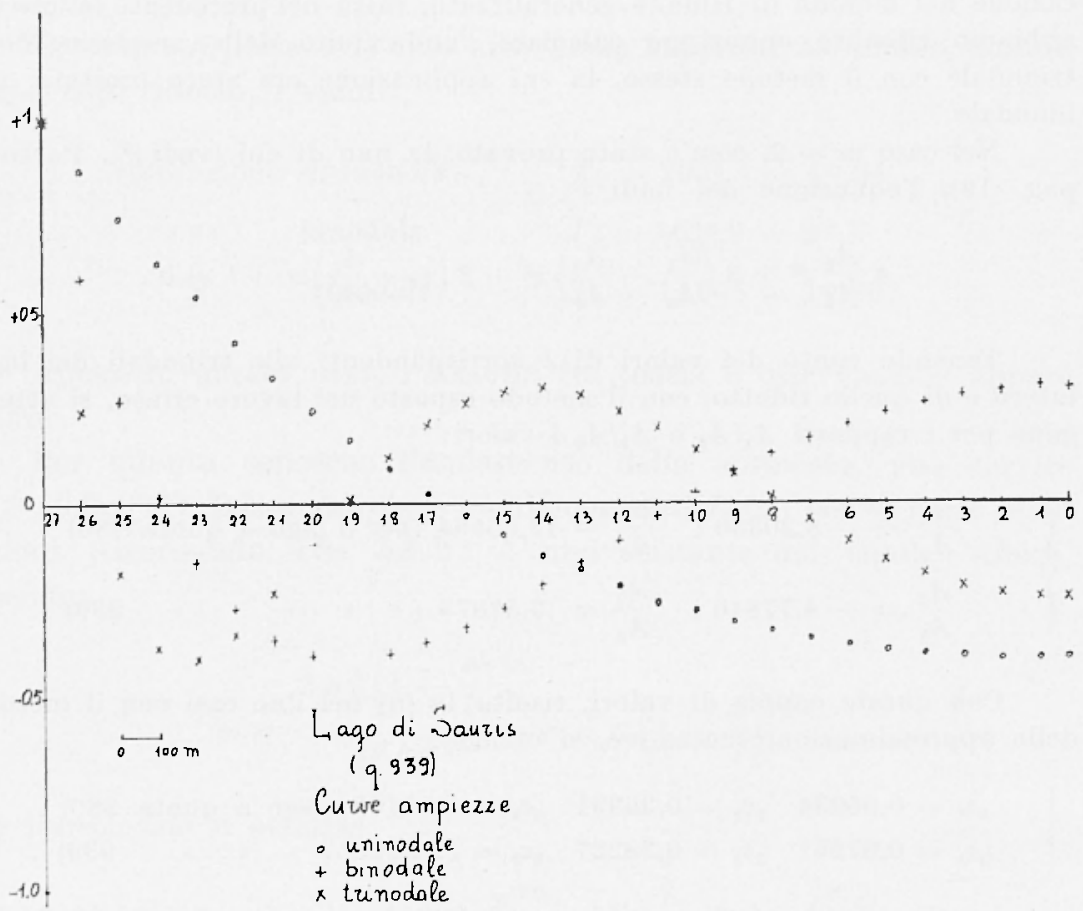

Fig. 22 
Com'era da attendersi, la posizione dei nodi, ottenuta con questo metodo, differisce alquanto da quella fornita dall'applicazione del metodo di Hidaka. Ciò si spiega col fatto che, specie per il lago completo, la parte orientale del bacino differisce notevolmente dal caso ideale, a motivo delle numerose, ampie insenature.

5. - Conclusioni. - L'osservazione conferma l'attendibilità dei risultati del calcolo, che, per quanto riguarda i periodi, ha condotto a valori pressochè coincidenti, pur nella diversità dei metodi usati.

Tenuto conto della superficie esterna dei laghi completo e ridotto, si può ritenere che - approssimativamente - nel passaggio dal lago completo al lago ridotto, il bacino ha perduto circa 52 milioni di $\mathrm{m}^{3}$ d'acqua (dei 70 milioni a bacino pieno). Il periodo dell'uninodale è diminuito da $6^{\mathrm{m}}, 2$ a $4^{\mathrm{m}}, 9$ circa. Non è detto che ciò debba necessariamente verificarsi sempre. Infatti, come mostra la teoria, i periodi sono direttamente proporzionali alla lunghezza e inversamente proporzionali alla radice quadrata della profondità del bacino. Prescindono cioè dall'entità della massa liquida. Una diminuzione di questa può addirittura condurre ad un bacino ridotto con periodo maggiore del corrispondente bacino intero: basta che la diminuzione di $h$ sia proporzionalmente maggiore di quella di $l$.

Nel caso specifico, e nell'ipotesi della validità del secondo metodo, il rapporto dei periodi dell'uninodale, per lago completo $\left(l_{\ell}\right)$ e lago ridotto $\left(l_{r}\right)$ vale:

$$
\frac{l_{i}}{l_{r}} \frac{\sqrt{h_{r}}}{\sqrt{h_{t}}}=\frac{3970}{2670} \frac{\sqrt{89}}{\sqrt{130}}=1,23
$$

del lago; per il lago a q. 939 fra le sezioni 4-5, 13-14, 21-22, alle distanze di $\mathrm{km} 0,45,1,37,2,12$ rispettivamente, sempre contate dall estremo Ovest del lago (Fig. 1).

J'er quanto concerne l'andamento delle ampiezze, esso si deduce formalmente - nel caso $m=2$ - dal $1^{0}$ membro delle [a]:

$$
\left\{\begin{array}{l}
\zeta^{\prime \prime \prime}=48,22256 z^{3}-60,77682 z^{2}+18,40660 z-1 \text { (lago a quota 980) } \\
\left.\zeta^{\prime \prime \prime}=42,90100 z^{3}-55,54857 z^{2}+17,58188 z-1 \text { (" " " } 939\right),
\end{array}\right.
$$

sostituendo alle $z \mathrm{i}$ valori che esse assumono nelle varie sezioni.

Is prima riga delle $[\beta]$ contiene gli zeri per $\zeta^{\prime \prime \prime}$ a q. 980, mentre la seconda contiene gli zeri per $\zeta^{\prime \prime \prime}$ a q. 939.

I risultati dei calcoli sono riassunti nella Tabella III, mentre le Figrg. 23 e 24 ne forniscono il grafieo. 
(he coincide appunto con il rapporto $6,1 / 4,94$ dei rispettivi valori calcolati (ed osservati). In ciò si ha una nuova riprova della validità delle teorie idrodinamiche, di cui ci si è serviti.

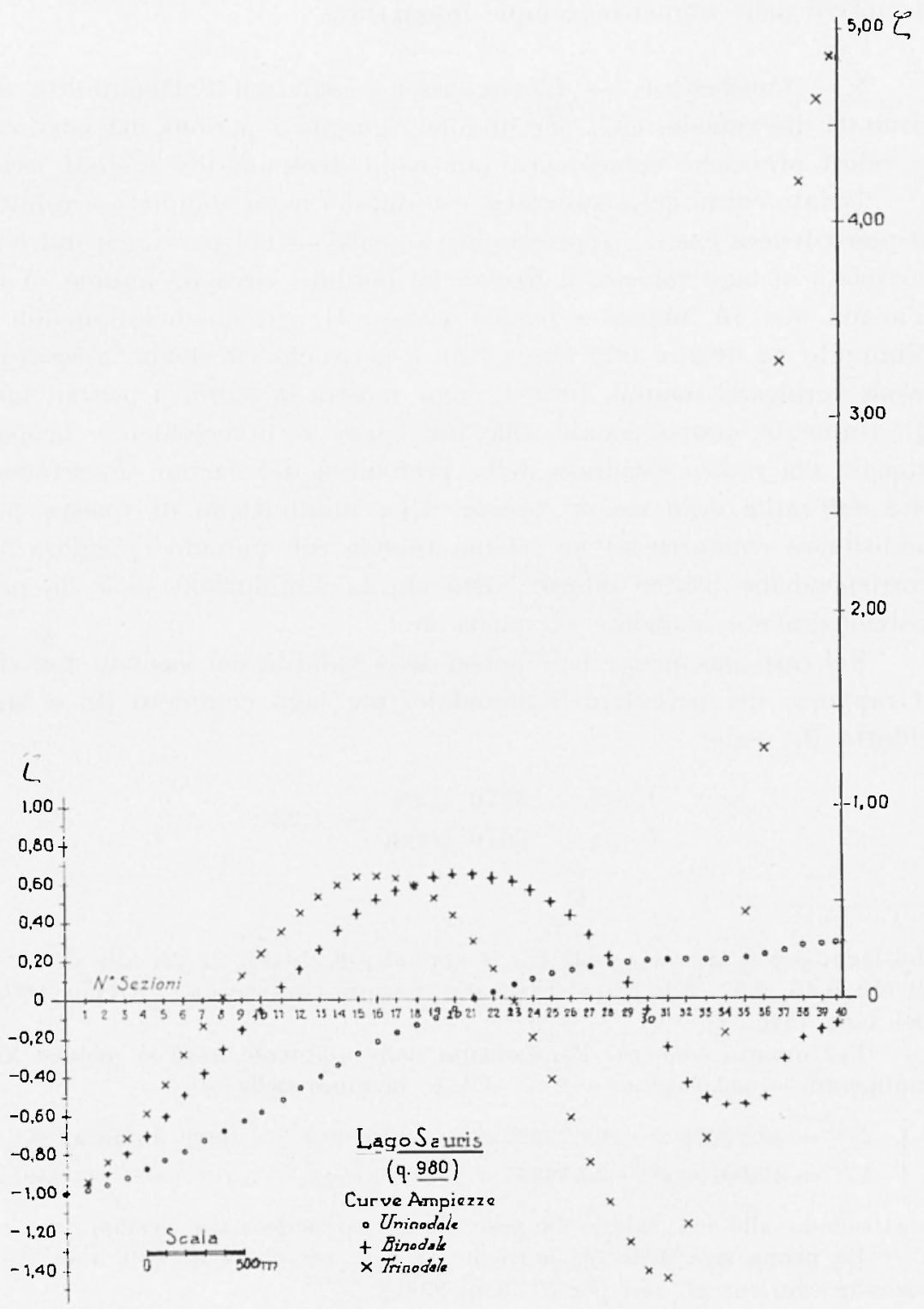

Fig. 23 


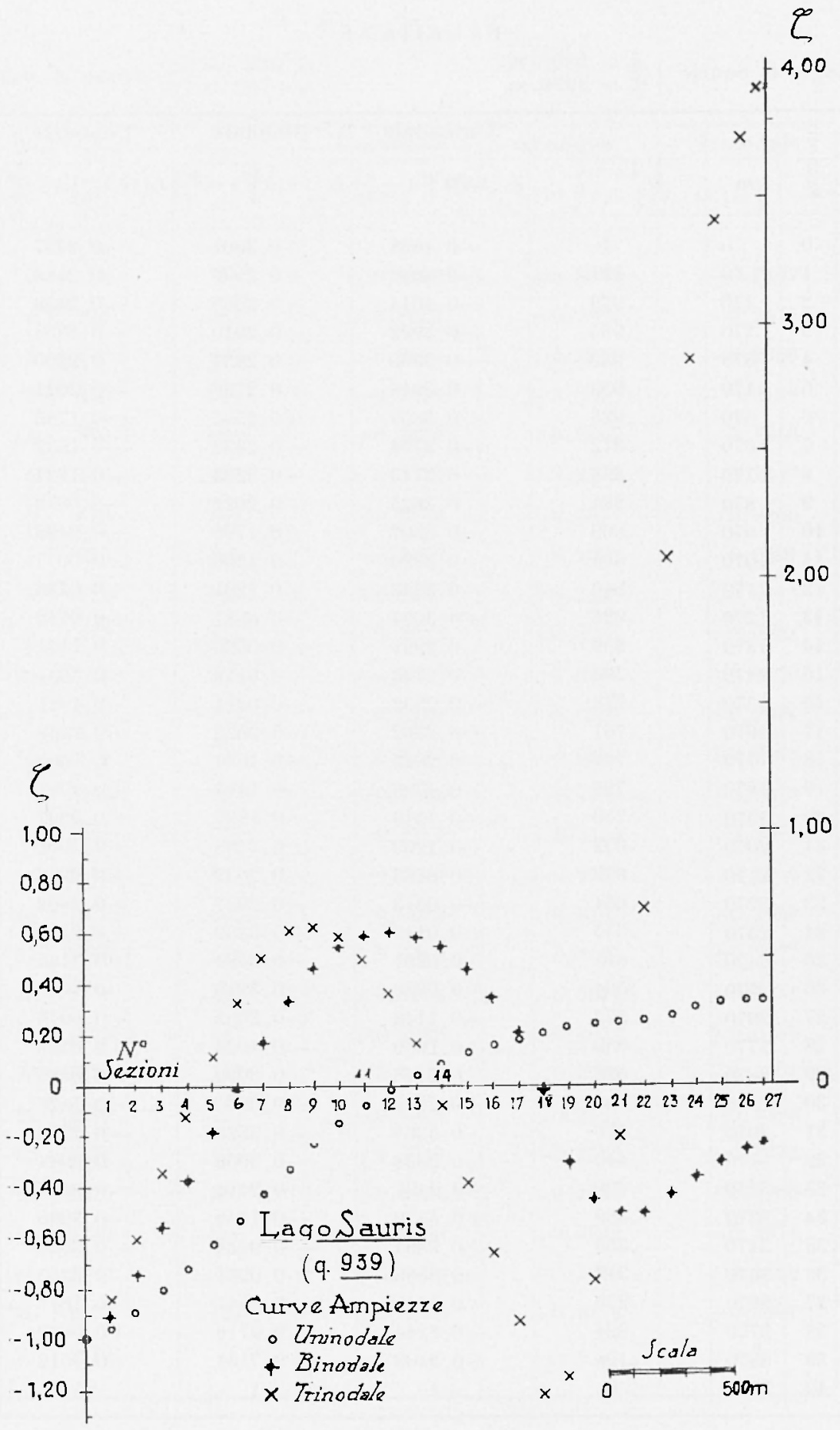

Fig. 24 
Taleella I

Lago di Sanris $\quad q=y \times u \quad m$

\begin{tabular}{|c|c|c|c|c|c|}
\hline$\Xi$ & $x$ & & Uninodale & Binodale & Trinodale \\
\hline कू. & $m$ & $\sqrt{1-\frac{\infty}{l}}$ & $J_{o}\left(3,\left.832\right|^{\prime} \overline{1-\frac{x}{l}}\right.$ & $J_{o}\left(\tau, 016 \sqrt{1-\frac{x}{l}}\right)$ & $J_{o}\left(10,173 / \overline{1-\frac{x}{l}}\right)$ \\
\hline 0 & 0 & 1 & $-0,4028$ & $-0,3001$ & $-0,2497$ \\
\hline I & 70 & .991 & $-0,4026$ & $-0,2996$ & $-0,2486$ \\
\hline 2 & 170 & .979 & $-0,4014$ & $+0,2969$ & $-0,2439$ \\
\hline 3 & 270 & .965 & $-0,3992$ & $-0,2910$ & $-0,2338$ \\
\hline 4 & 370 & .952 & $-0,3960$ & $\therefore 0,2831$ & $-0,2200$ \\
\hline 5 & 470 & .939 & $-0,3918$ & $+0,2726$ & $-0,2021$ \\
\hline (j) & 570 & .925 & $-0,3859$ & $+0,2585$ & $-0,1786$ \\
\hline 7 & 670 & .912 & $-0,3794$ & $\div 0,2433$ & $-0,1532$ \\
\hline 8 & 770 & .898 & $-0,3713$ & $-0,2238$ & $-0,1224$ \\
\hline 9 & 870 & .884 & $-0,3625$ & $-0,2022$ & $-0,0872$ \\
\hline 10 & 970 & .869 & $-0,3507$ & $-0,1765$ & $--0,0498$ \\
\hline 11 & 1070 & .855 & $-0,3390$ & $+0,1506$ & $--0,0077$ \\
\hline 12 & 1170 & .840 & $-0,3253$ & $\div 0,1201$ & $+0,0296$ \\
\hline 13 & 1270 & .825 & $-0,3094$ & $+0,0881$ & $+0,0712$ \\
\hline 14 & 1370 & .809 & $-0,2921$ & $+0,0520$ & $\div 0,1144$ \\
\hline 15 & 1470 & .794 & $-0,2743$ & $+0,0172$ & $-0,1531$ \\
\hline 16 & 1570 & .778 & $-0,2532$ & $-0,0211$ & $+0,1911$ \\
\hline 17 & 1670 & .761 & $-0,2302$ & $-0,0622$ & $-0,2268$ \\
\hline 18 & 1770 & .744 & $-0,2055$ & $-0,1034$ & $+0,2566$ \\
\hline 19 & 1870 & .727 & $-0,1793$ & $-0,1443$ & $+0,2791$ \\
\hline 20 & 1970 & .710 & $-0,1516$ & $-0,1837$ & $+0,2937$ \\
\hline 21 & 2070 & .692 & $-0,1209$ & $-0,2238$ & $+0,3000$ \\
\hline 22 & 2170 & .673 & $-0,0783$ & $-0,2632$ & $-0,2958$ \\
\hline 23 & 2270 & .6554 & $-0,0513$ & $-0,2987$ & $\therefore 0,2802$ \\
\hline 24 & 2370 & .635 & $-0,0145$ & $-0,3309$ & $-0,2537$ \\
\hline 25 & 2470 & .615 & $+0,0251$ & $-0,3584$ & $-0,2145$ \\
\hline 26 & 2570 & .594 & $-0,0686$ & $-0,3810$ & $+0,1623$ \\
\hline 27 & 2670 & .572 & $-0,1148$ & $-0,3962$ & $-0,0976$ \\
\hline 28 & 2770 & .550 & 0,1609 & $-0,4026$ & $+0,0253$ \\
\hline 29 & 2870 & .526 & $+0,2146$ & $-0,3990$ & $-0,0585$ \\
\hline 30 & 2970 & .502 & $+0,2679$ & $-0,3830$ & $-0,1420$ \\
\hline 31 & 3070 & .476 & $-0,3261$ & $-0,3526$ & $-0,2276$ \\
\hline 32 & 3170 & .449 & $+0,3858$ & $-0,3066$ & $-0,3044$ \\
\hline 33 & 3270 & .420 & $+0,4497$ & $-0,2414$ & $--0,3656$ \\
\hline 34 & 3370 & .389 & $+0,5170$ & $-0,1550$ & $--0,3996$ \\
\hline 35 & 3470 & .355 & $+0,5884$ & $-0,0434$ & $-0,3928$ \\
\hline 36 & 3570 & .317 & $+0,6586$ & $-1-0,0971$ & $-0,3265$ \\
\hline 37 & 3670 & .275 & $+0,7410$ & $+0,2647$ & $-0,1841$ \\
\hline 38 & 3770 & .224 & $+0,8244$ & $+0,4716$ & $+0,0670$ \\
\hline 39 & 3870 & .198 & 0,9103 & $+0,7154$ & $+0,4512$ \\
\hline 40 & 3970 & 0 & -1 & $i-1$ & +1 \\
\hline
\end{tabular}


$\mathrm{T} a \mathrm{bella}$ I I

Lago di Sauris $\quad\left\{\begin{array}{r}q=939 \mathrm{~m} \\ l=2670 \mathrm{~m}\end{array}\right.$

\begin{tabular}{|c|c|c|c|c|c|}
\hline$\Xi$ & $x$ & & Uninodale & Binodale & 'Trinodale \\
\hline 芯 & $m$ & $11-\frac{\infty}{l}$ & $J_{o}(3,832 \sqrt{1-x})$ & $J_{o}\left(7,016 \sqrt{1-\frac{x}{l}}\right.$ & $J_{0}\left(10,173 / \sqrt{1-\frac{x}{l}}\right)$ \\
\hline 0 & 0 & 1 & $-0,4028$ & $+0,3001$ & $-0,2497$ \\
\hline 1 & 70 & .987 & $-0,4023$ & $+0,2988$ & $-0,2475$ \\
\hline 2 & 170 & .967 & $-0,3994$ & $-1-0,2921$ & $-0,2356$ \\
\hline 3 & 270 & .948 & $-0,3947$ & $+0,2800$ & $-0,2149$ \\
\hline 4 & 370 & .928 & $-0,3872$ & $-0,2617$ & $-0,1840$ \\
\hline 5 & 470 & .908 & $-0,3773$ & $+0,2377$ & $-0,1447$ \\
\hline 6 & 570 & .887 & $-0,3643$ & $+0,2071$ & $-0,0960$ \\
\hline 7 & 670 & .865 & $-0,3475$ & $+0,1692$ & $-0,0391$ \\
\hline 8 & 770 & .844 & $-0,3287$ & $+0,1283$ & $+0,0185$ \\
\hline 9 & 870 & .821 & $-0,3055$ & $-0,0791$ & 0,0821 \\
\hline 10 & 970 & .798 & $-0,2745$ & $+0,0266$ & $-0,1430$ \\
\hline 11 & 1070 & .774 & $-0,2544$ & $-0,0309$ & $-1-0,2001$ \\
\hline 12 & 1170 & .750 & $-0,2145$ & $-0,0889$ & $+0,2468$ \\
\hline 13 & 1270 & .724 & $-0,1708$ & $-0,1512$ & $+0,2823$ \\
\hline 14 & 1370 & .698 & $-0,1313$ & $-0,2115$ & $-\infty 0,2991$ \\
\hline 15 & 1470 & .671 & $-0,0832$ & $-0,2671$ & $+0,2946$ \\
\hline 16 & 1570 & .642 & $-0,0283$ & $-0,3195$ & $+0,2633$ \\
\hline 17 & 1670 & .612 & $+0,0313$ & $-0,3621$ & $+0,2077$ \\
\hline 18 & 1770 & .581 & $+0,0958$ & $-0,3911$ & $+-0,1251$ \\
\hline 19 & 1870 & .547 & +0.1688 & $-0,4027$ & $+0,0] 51$ \\
\hline 20 & 1970 & .512 & $+0,2458$ & $-0,3911$ & $-0,1074$ \\
\hline 21 & 2070 & .474 & +0.3305 & $-0,3497$ & $-0,2338$ \\
\hline 22 & 2170 & .433 & $+0,4210$ & $-0,2726$ & $-0,3413$ \\
\hline 23 & 2270 & .387 & $+0,5424$ & $-0,1491$ & $-0,4005$ \\
\hline 24 & 2370 & .336 & $+0,6266$ & $+0,0249$ & $-0,3674$ \\
\hline 25 & 2470 & .274 & $+0,7428$ & $+0,2688$ & $-0,1799$ \\
\hline 26 & 2570 & .194 & $-\div 0,8665$ & $-0,5878$ & $+0,2391$ \\
\hline 27 & 2670 & 0 & +1 & +1 & +1 \\
\hline
\end{tabular}


Ta be Ila I I I

\begin{tabular}{|c|c|c|c|c|c|}
\hline \multicolumn{6}{|c|}{ ' $\mathrm{R}$ I N O D A L L } \\
\hline \multicolumn{3}{|c|}{ Quota 980} & \multicolumn{3}{|c|}{ Quota 939} \\
\hline Sezioni & $\begin{array}{l}\Delta x \\
m\end{array}$ & $\zeta^{\prime \prime \prime}$ & Sezioni & $\begin{array}{l}\Delta x \\
m\end{array}$ & $\zeta^{\prime \prime \prime}$ \\
\hline 0 & () & -1 & 0 & 0 & -1 \\
\hline 1 & 100 & -0.95774 & 1 & 100 & -0.84934 \\
\hline 2 & 200 & -0.83534 & 2 & 200 & -0.60702 \\
\hline 3 & 300 & -0.69685 & 3 & 300 & -0.33349 \\
\hline 4 & 400 & -0.58882 & 4 & 400 & -0.11712 \\
\hline$\tilde{5}$ & 500 & -0.45077 & 5 & 500 & -0.11527 \\
\hline 6 & 600 & -0.27581 & 6 & 600 & +0.32914 \\
\hline 7 & 700 & -0.13217 & 7 & 700 & -0.50396 \\
\hline 8 & 800 & -0.00610 & 8 & 800 & -0.60764 \\
\hline 9 & 900 & +0.12563 & 9 & 900 & -0.63953 \\
\hline 10 & 1000 & +0.24401 & 10 & 1000 & -0.59475 \\
\hline II & 1100 & 0.35790 & 11 & 1100 & +0.49999 \\
\hline 12 & 1200 & -0.45365 & 12 & 1200 & -0.36905 \\
\hline 13 & 1300 & -0.53915 & 13 & 1300 & +0.16967 \\
\hline 14 & 1400 & +0.59590 & 14 & 1400 & $-0.070+0$ \\
\hline 15 & 1500 & +0.62867 & 15 & 1500 & $-0.3782 \mathrm{I}$ \\
\hline 16 & 1600 & +0.63537 & 16 & 1600 & -0.66052 \\
\hline 17 & 1700 & +0.62023 & 17 & 1700 & -0.92631 \\
\hline 18 & 1800 & 0.58536 & 18 & 1800 & -1.22304 \\
\hline 19 & 1900 & +0.51920 & 19 & 1900 & -1.16364 \\
\hline 20 & 2000 & +0.42460 & 20 & 2000 & -0.77446 \\
\hline 21 & 2100 & +0.30044 & 21 & 2100 & -0.19622 \\
\hline 22 & 2200 & +0.15744 & 22 & 2200 & +0.69452 \\
\hline 23 & 2300 & -0.00890 & 23 & 2300 & +2.08029 \\
\hline 24 & 2400 & -0.20412 & 24 & 2400 & -12.86468 \\
\hline 25 & 2500 & -0.40884 & 25 & 2500 & +-3.41056 \\
\hline 26 & 2600 & -0.61385 & 26 & 2600 & +3.73307 \\
\hline 27 & 2700 & -0.83853 & 27 & 2670 & +3.93431 \\
\hline 28 & 2800 & -1.05293 & & & \\
\hline 29 & 2900 & -1.25052 & & & \\
\hline 30 & 3000 & -1.41945 & & & \\
\hline 31 & 3100 & -1.45233 & & & \\
\hline 32 & 3200 & -1.17453 & & & \\
\hline 33 & 3300 & -0.71450 & & & \\
\hline 34 & 3400 & -0.16979 & & & \\
\hline .35 & 3500 & -0.45439 & & & \\
\hline 36 & 3600 & +1.29837 & & & \\
\hline 37 & 3700 & +3.19061 & & & \\
\hline 38 & 3800 & +4.21746 & & & \\
\hline 39 & 3900 & +4.63543 & & & \\
\hline 40 & 3970 & $-1-4.85234$ & & & \\
\hline
\end{tabular}


Qualora il rapporto $\frac{l_{i}}{l_{r}} \frac{\sqrt{h_{r}}}{\sqrt{h_{i}}}$ risultasse inferiore all'unità (ciò che avverrebbe eflettivamente per $l_{r}=l_{i}$ ), allora alla diminuzione del livello nel bacino corrisponderebbe un aumento del periodo delle oscillazioni proprie della massa d'acqua.

Poiché i bacini naturali in genere, e quelli artificiali in particolare, col tempo, vamno lentamente insabbiandosi per il continuo apporto del materiale d'erosione trasportato dagli immissari, a parità di livello e di forma della superficie esterna, ci si deve attendere un progressivo aumento dei periodi propri dei bacini lacustri.

\section{BIBLIOGRAFIA}

(1) Ronualdi G., Spadea M. C., Studio preliminare sulle oscillazioni libere del Lago di Sauris a due diverse quote. "Annali li Geofisica ", XIX, 4, (1966).

(2) Calor P., Le sesse del Lago di Garda. Parte I. "Annali di Geofisica" I, I (1948); Parte II, ivi, vol. I, 2, (1948).

(3) Cinrstat G., On the hydrodynamical Theory of Seiches. "Transactions of the Royal Society of Edinburg ", XLI (1905), XLV (1906), XLVI (1907-8).

(1) Sansone G., Equazioni differenziali nel campo reale. Parte I, p. 159. Nicola Zanichelli Editore (1941).

(5) Sassone G., Equazioni differenziali nel campo reale. Parte I, p. 164, Nicola Zanichelli Editore (1941). 